\title{
Inefficiency of Games with Social Context
}

\author{
Aris Anagnostopoulos • Luca Becchetti . \\ Bart de Keijzer • Guido Schäfer
}

Received: date / Accepted: date

\begin{abstract}
The study of other-regarding player behavior such as altruism and spite in games has recently received quite some attention in the algorithmic game theory literature. Already for very simple models, it has been shown that altruistic behavior can actually be harmful for society in the sense that the price of anarchy may increase as the players become more altruistic. In this paper, we study the severity of this phenomenon for more realistic settings in which there is a complex underlying social structure, causing the players to direct their altruistic and spiteful behavior in a refined player-specific sense (depending, for example, on friendships that exist among the players). Our findings show that the increase in the price of anarchy is modest for congestion games and minsum scheduling games, whereas it might be drastic for generalized second price auctions.
\end{abstract}

\section{Introduction}

Many practical situations involve a group of strategic decision makers who attempt to achieve their own self-interested goals. It is well known that strategic

This research was partially supported by the EU FET projects MULTIPLEX no. 317532 and SIMPOL no. 610704, the ERC StG Project PAAI 259515, the FET-Open FOC no. 255987, and the Google Research Award for Economics and Market Algorithms.

Aris Anagnostopoulos, Luca Becchetti

Sapienza University of Rome, Italy

E-mail: \{aris,becchetti\}@dis.uniroma1.it

Bart de Keijzer

Sapienza University of Rome, Italy, and

CWI and VU University of Amsterdam, The Netherlands

E-mail: dekeijzer@dis.uniroma1.it

Guido Schäfer

CWI and VU University Amsterdam, The Netherlands

E-mail: g.schaefer@cwi.nl 
decision making may result in outcomes that are suboptimal for the society as a whole. The need to gain an accurate understanding of the extent of suboptimality caused by selfish behavior has led to the study of the inefficiency of equilibria in algorithmic game theory. In this context, a common inefficiency measure is the price of anarchy [27], which relates the worst-case cost of a Nash equilibrium to the one of an optimal outcome.

More recently, quite some attention has been given to more general settings in which the players do not necessarily behave entirely selfishly, but may alternatively exhibit spiteful or altruistic behavior; see, for instance, $[4,6,7,10$, $12,14,19,22-24]$. Studying such alternative behaviors in games is motivated by the observation that altruism and spite are phenomena that frequently occur in real life (see, e.g., [20]). Consequently, it is desirable to incorporate such alternative behavior in game-theoretical analyses.

Previous work on the price of anarchy for spiteful and altruistic games has focused on simple models of spite and altruism, where a spite/altruism level $\alpha_{i}$ is associated to each player $i$ denoting the extent to which his perceived cost is influenced by any nonspecific other player. Already for these simple models it has been observed in a series of papers $[7,10,12]$ that altruistic behavior can actually be harmful in the sense that the price of anarchy may increase as players become more altruistic. For example, Chen et al. [12] show that for congestion games with linear latency functions and uniform players (i.e., $\alpha_{i}=\alpha \in[0,1]$ for every $\left.i\right)$ the price of anarchy is $\frac{5+4 \alpha}{2+\alpha}$ and this bound is tight. In particular, the price of anarchy degrades from 2.5 in the purely selfish setting $(\alpha=0)$ to 3 in the fully altruistic setting $(\alpha=1)$.

This observation served as a starting point for the investigations conducted in this paper. The main question that we address here is: How severe can this effect be if one considers more refined models of altruism that capture complex social relationships between the players?

Our Contributions. In this paper, we study a more general player-specific model of spite and altruism. Our model can be viewed as extending a given strategic game by imposing a social-network structure on top of the players, which specifies for each pair of players $(i, j)$ an altruism/spite level $\alpha_{i j}$ signifying how much player $i$ cares about player $j$; these relations are not necessarily symmetric. This allows us to model more realistic settings in which the behavior of the players depends on a complex underlying social structure, expressing friendships and animosities among the players. Our altruistic games fall into the framework of social context games proposed by Ashlagi et al. [2].

For this general model of games with altruism and spite, we are interested in studying the price of anarchy. The smoothness framework, originally introduced by Roughgarden [33], has become a standard method for proving upper bounds on the price of anarchy. Basically, this framework shows that such bounds can be derived by establishing a certain smoothness condition. An additional strength of this approach is that the smoothness condition allows to derive upper bounds on the price of anarchy (also called robust price of anarchy in this context) for various solution concepts, ranging from pure Nash 


\begin{tabular}{|l|c|c|}
\hline Game & POA (selfish) & POA (altruistic) \\
\hline \hline linear congestion game & $\frac{5}{2}[3,15]$ & 7 \\
load balancing game & $\approx 2.06[34,8]$ & $\approx 4.24$ \\
related machine scheduling game & $2[25]$ & $\approx 7.46$ \\
unrelated machine scheduling game & $4[17]$ & $\approx 23.31$ \\
generalized second price auction & $\approx 2.31[29,28,9,11]$ & $2(n+1)$ \\
\hline
\end{tabular}

Table 1 Upper bounds on the coarse correlated price of anarchy of altruistic games derived in this paper in comparison to the purely selfish setting.

equilibria to coarse correlated equilibria; the latter being naturally related to outcomes resulting from natural learning algorithms (e.g., see [37]). Here, we extend the smoothness framework such that it can be used conveniently to study games with social context.

Using this extension, we prove upper bounds on the price of anarchy for altruistic versions of three classes of well-studied games: congestion games, minsum scheduling games, and generalized second price auctions. We show that for unrestricted altruism levels the price of anarchy is unbounded. In particular, this happens if there is a player $i$ who cares more about some friend $j$ than about himself, i.e., $\alpha_{i j}>\alpha_{i i}$. We, therefore, derive our upper bounds under the mild assumption that each player cares positively about himself and he cares about any other player at most as much as he cares about himself; we refer to this as restricted altruistic social context. Under this assumption, we derive the following upper bounds on the coarse correlated price of anarchy:

- A bound of 7 for altruistic linear congestion games, and a bound of $\varphi^{3} \approx$ 4.24 for the special case of load balancing games, where $\varphi=(1+\sqrt{5}) / 2$ denotes the golden ratio.

- A bound of $4+2 \sqrt{3} \approx 7.46$ and $12+8 \sqrt{2} \approx 23.31$ for altruistic minsum machine scheduling games for related and unrelated machines, respectively.

- A bound of 2(n+1) for altruistic generalized second price auctions, where $n$ is the number of players.

Our results therefore show that for congestion games and minsum scheduling games the price of anarchy cannot increase drastically; specifically, it remains constant, independently of how complex the underlying altruistic social context is. On the other hand, for generalized second price auctions our results seem to suggest that the price of anarchy may degrade drastically: we obtain and upper bound of $2(n+1)$, as opposed to a small constant in the purely selfish setting [9]. We also prove that this bound is asymptotically tight if one resorts to the smoothness approach; i.e., in order to improve on our bound one needs to come up with more sophisticated techniques that go beyond the standard smoothness notion. A summary of the bounds derived in this paper is given in Table 1. 
Related Work. There are several papers that propose models of altruism and spite $[6,7,10,12,14,19,22-24]$. All these models are special cases of the one studied here. Among these articles, the inefficiency of equilibria in the presence of altruistic/spiteful behavior was studied for various games in $[7,10,12,14$, 19]. After its introduction by Roughgarden [33], the smoothness framework has been adapted in various directions $[31,35,36]$, including an extension to a particular model of altruism by Chen et al. [12], which constitutes a special case of the altruistic games considered here.

Biló et al. [4] also studied social context congestion games, in the case where the perceived cost of a player is the minimum, maximum, or sum of the immediate cost of his neighbors. They establish, among other results, an upper bound of $17 / 3$ on the pure price of anarchy of linear congestion games for a special case of the setting we study here.

Related but different from our setting, is the concept of graphical congestion games $[5,21]$. Here the cost and the strategy set of a player depends only on a subset of the players.

After publication of a preliminary conference version of the present paper [1], follow-up work by Rahn and Schäfer appeared as [30]. In their paper, the authors relate the study of altruistic extensions of games to a class of games, named social contribution games, and improve some of the upper bounds we give here. In particular, they show that the coarse correlated price of anarchy is $17 / 3$ for linear congestion games and 4 for unrelated machine scheduling games; both bounds are tight.

\section{Preliminaries}

Altruistic Extensions of Games. We study the effect of altruistic behavior in strategic games. To model the complex relationships between the players, we equip the underlying game with a social context. More precisely, let $\Gamma=$ $\left(N,\left\{\Sigma_{i}\right\}_{i \in N},\left\{c_{i}\right\}_{i \in N}\right)$ be a strategic game (termed base game), where $N=$ $\{1, \ldots, n\}$ is the set of players, $\Sigma_{i}$ is the strategy set of player $i$, and $c_{i}$ : $\Sigma \rightarrow \mathbb{R}$ is the direct cost function of player $i$ that maps every strategy profile $s \in \Sigma=\Sigma_{1} \times \cdots \times \Sigma_{n}$ to a real value. Unless stated otherwise, we assume that $\Gamma$ is a cost minimization game, i.e., every player $i$ wants to minimize his individual cost function $c_{i}$. Further, we assume that a social context is given by an $n \times n$ matrix $\alpha \in \mathbb{R}^{n \times n}$.

Given a base game $\Gamma$ and a social context $\alpha$, the $\alpha$-extension of $\Gamma$ is defined as the strategic game $\Gamma^{\alpha}=\left(N,\left\{\Sigma_{i}\right\}_{i \in N},\left\{c_{i}^{\alpha}\right\}_{i \in N}\right)$, where for all $i \in N$ and $s \in \Sigma$, the perceived cost $c_{i}^{\alpha}(s)$ of player $i$ is given by

$$
c_{i}^{\alpha}(s)=\sum_{j=1}^{n} \alpha_{i j} c_{j}(s) .
$$

Thus, the perceived cost of player $i$ in the $\alpha$-extension is the $\alpha_{i j}$-weighted sum of the individual direct costs of all players in the base game. A positive 
(negative) $\alpha_{i j}$ value signifies that player $i$ cares positively (negatively) about the direct cost of player $j$, which can be interpreted as an altruistic (spiteful) attitude of $i$ towards $j$. Note that $\alpha_{i i}$ specifies how player $i$ cares about himself; we also call $\alpha_{i i}$ the self-perception level. For simplicity, we will often refer to the resulting game $\Gamma^{\alpha}$ as the $\alpha$-extension, without explicitly mentioning the base game $\Gamma$ and the social context $\alpha$.

The above viewpoint has a natural interpretation in terms of social networks: Suppose the players in $N$ are identified with the nodes of a complete directed graph $G=(N, A)$. The weight of an edge $(i, j) \in A$ is equal to $\alpha_{i j}$, specifying the extent to which player $i$ cares about the cost of player $j$.

Even though the smoothness notion that we introduce below applies to arbitrary $\alpha$-extensions of games, the main focus of this paper is on altruistic behavior. We distinguish between unrestricted and restricted altruistic social contexts $\alpha$. In the unrestricted case we assume that $\alpha_{i j} \geq 0$ for every $i, j \in N$; in particular, the self-perception level of a player can be zero. In this case, one can prove trivial lower bounds for the price of anarchy, just by setting $\alpha_{i j}=0$, for all $i, j$. For this reason we consider also the more interesting restricted case. In the restricted case, every player has a positive self-perception level and cares about himself at least as much as about any other player, namely, $\alpha_{i i}>0$ and $\alpha_{i i} \geq \alpha_{i j} \geq 0$ for every $i, j \in N, i \neq j$. In the latter case, we can normalize $\alpha$ without loss of generality such that $\alpha_{i i}=1$ for every player $i{ }^{1}$ Given an altruistic social context $\alpha$, we refer to the resulting $\alpha$-extension also as $\alpha$-altruistic game.

Coarse Correlated Equilibria and the Price of Anarchy. We are interested in the efficiency loss caused by altruistic behavior. Let $C: \Sigma \rightarrow \mathbb{R}$ be a social cost function that maps strategy profiles to real numbers. Most of the time in this paper, the social cost will refer to the sum of the direct costs of all players, namely, $C(s)=\sum_{i=1}^{n} c_{i}(s)$. The motivation is that we are interested in the efficiency of the outcome resulting from altruistic behavior, which is modeled through the altered perceived cost functions. We use $s^{*}$ to refer to an optimal strategy profile that minimizes $C$, i.e., $C\left(s^{*}\right) \leq C(s)$ for every $s \in \Sigma$.

We focus on the inefficiency of coarse correlated equilibria, which are defined as follows (e.g., see [37]): Let $\sigma$ be a probability distribution over $\Sigma$ and let $\sigma_{-i}$ denote the projection of $\sigma$ onto

$$
\Sigma_{-i}=\Sigma_{1} \times \cdots \times \Sigma_{i-1} \times \Sigma_{i+1} \times \cdots \times \Sigma_{n} .
$$

Then $\sigma$ is a coarse correlated equilibrium of the $\alpha$-extension $\Gamma^{\alpha}$ if, for every player $i$ and every strategy $s_{i}^{*} \in \Sigma_{i}$, it holds that

$$
\mathbf{E}_{s \sim \sigma}\left[c_{i}^{\alpha}(s)\right] \leq \mathbf{E}_{s_{-i} \sim \sigma_{-i}}\left[c_{i}^{\alpha}\left(s_{i}^{*}, s_{-i}\right)\right] .
$$

We use $C C E\left(\Gamma^{\alpha}\right)$ to denote the set of coarse correlated equilibria of $\Gamma^{\alpha}$. Coarse correlated equilibria include several other solution concepts, such as correlated

\footnotetext{
1 To see this, note that, by dividing all $\alpha_{i j}$ by $\alpha_{i i}>0$, the set of equilibria and the social cost of any outcome remain the same.
} 
equilibria, mixed Nash equilibria, and pure Nash equilibria (e.g., see [33]), and are thus guaranteed to exist for finite games.

We study the price of anarchy [27] of coarse correlated equilibria. For an $\alpha$-extension $\Gamma^{\alpha}$, define

$$
P O A\left(\Gamma^{\alpha}\right)=\sup _{s \in C C E\left(\Gamma^{\alpha}\right)} \frac{C(s)}{C\left(s^{*}\right)},
$$

where $s^{*}$ is a strategy profile that minimizes $C$. The coarse correlated price of anarchy of a class of $\alpha$-extensions $\mathcal{G}$ is defined as

$$
\operatorname{POA}(\mathcal{G})=\sup _{\Gamma^{\alpha} \in \mathcal{G}} \operatorname{POA}\left(\Gamma^{\alpha}\right) .
$$

Some material has been omitted from the main body of the paper and can be found in the appendix.

\section{Smoothness}

Smoothness. Roughgarden [33] introduced a general smoothness framework to derive bounds on the coarse correlated price of anarchy. This framework applies to strategic games with sum-bounded social cost functions. In the context of $\alpha$-extensions of games this requirement is equivalent to $C(s) \leq \sum_{i=1}^{n} c_{i}^{\alpha}(s)$ for every $s \in \Sigma$. Note that this condition is not necessarily satisfied if we allow arbitrary social contexts $\alpha \in \mathbb{R}^{n \times n}$. Next we extend this framework to $\alpha$-extensions with arbitrary social contexts and social cost functions.

Definition 1 Let $\Gamma^{\alpha}$ be an $\alpha$-extension of a cost minimization game with $\alpha \in \mathbb{R}^{n \times n}$ and social cost function $C$. Further, let $s^{*}$ be an optimal strategy profile with respect to $C . \Gamma^{\alpha}$ is $(\lambda, \mu)$-smooth if for every strategy profile $s \in \Sigma$ it holds that

$$
\sum_{i=1}^{n} \sum_{j=1}^{n} \alpha_{i j}\left(c_{j}\left(s_{i}^{*}, s_{-i}\right)-c_{j}(s)\right) \leq \lambda C\left(s^{*}\right)+(\mu-1) C(s) .
$$

The following theorem shows that $(\lambda, \mu)$-smoothness implies a bound on the coarse correlated price of anarchy of $\alpha$-extensions.

Theorem 1 Let $\Gamma^{\alpha}$ be an $\alpha$-extension of a cost minimization game with $\alpha \in$ $\mathbb{R}^{n \times n}$ and social cost function $C$. If $\Gamma^{\alpha}$ is $(\lambda, \mu)$-smooth with $\mu<1$, then the coarse correlated price of anarchy of $\Gamma^{\alpha}$ is at most $\lambda /(1-\mu)$.

In the purely selfish setting (i.e., when $\alpha_{i i}=1$ and $\alpha_{i j}=0$ for every $i, j \in N, i \neq j$ ) our smoothness definition is slightly more general than the one in [33] where (2) is required to hold for any arbitrary strategy profile $s^{*}$. Also, in [33] the analogue of Theorem 1 is shown under the additional assumption that $C$ is sum-bounded. Here, we get rid of this assumption. The proof of Theorem 1 can be found in Appendix A. 
Efficiency of no-regret algorithms. The above smoothness definition allows us to import some additional results from [33]. In particular, it proves useful in the context of learning algorithms generating no-regret sequences.

Theorem 2 Let $\Gamma^{\alpha}$ be an $\alpha$-extension of a cost minimization game with $\alpha \in$ $\mathbb{R}^{n \times n}$ and social cost function $C$. Suppose that $s^{1}, \ldots, s^{T}$ is a sequence of outcomes in which every player experiences vanishing average external regret, i.e., for every player $i \in N$

$$
\frac{1}{T} \sum_{t=1}^{T} c_{i}^{\alpha}\left(s^{t}\right) \leq \frac{1}{T}\left[\min _{s_{i}^{\prime} \in \Sigma_{i}} \sum_{t=1}^{T} c_{i}^{\alpha}\left(s_{i}^{\prime}, s_{-i}^{t}\right)\right]+o(1) .
$$

If $\Gamma^{\alpha}$ is $(\lambda, \mu)$-smooth with $\mu<1$, then the average cost of the sequence is

$$
\frac{1}{T} \sum_{t=1}^{T} C\left(s^{t}\right) \leq \frac{\lambda}{1-\mu} C\left(s^{*}\right)+o(1) .
$$

The proof is similar to the one in [33] and can be found in Appendix A.

\section{Congestion Games}

In a congestion game $\Gamma=\left(N, E,\left\{d_{e}\right\}_{e \in E},\left\{\Sigma_{i}\right\}_{i \in N}\right)$ we are given a set of players $N=\{1, \ldots, n\}$, a set of facilities $E$ with a delay function $d_{e}: \mathbb{N} \rightarrow \mathbb{R}$ for every facility $e \in E$, and a strategy set $\Sigma_{i} \subseteq 2^{E}$ for every player $i \in N$. For a strategy profile $s \in \Sigma=\Sigma_{1} \times \cdots \times \Sigma_{n}$, define $x_{e}(s)$ as the number of players using facility $e \in E$, i.e., $x_{e}(s)=\left|\left\{i \in N: e \in s_{i}\right\}\right|$. The direct cost of player $i$ is defined as $c_{i}(s)=\sum_{e \in s_{i}} d_{e}\left(x_{e}(s)\right)$ and the social cost function is given by $C(s)=\sum_{i=1}^{n} c_{i}(s)$. In a linear congestion game, the delay function of every facility $e \in E$ is of the form $d_{e}(x)=a_{e} x+b_{e}$, where $a_{e}, b_{e} \in \mathbb{Q} \geq 0$ are nonnegative rational numbers.

We note that [22] studies conditions and algorithms for the existence of Nash equilibria in linear congestion games, for a special case of our model.

\subsection{General Linear Congestion Games}

Theorem 3 Every $\alpha$-altruistic extension of a linear congestion game with restricted altruistic social context $\alpha$ is $\left(\frac{7}{3}, \frac{2}{3}\right)$-smooth. Therefore, the coarse correlated price of anarchy is at most 7 for these games.

We need the following technical lemma for the proof of Theorem 3 .

Lemma 1 ([13]) For every two integers $x, y \in \mathbb{N}$

$$
(x+1) y+x y \leq \frac{7}{3} y^{2}+\frac{2}{3} x^{2} .
$$


The proof follows from Lemma 4.5 in [13] (with $\eta=1, \beta=0$ and $\gamma=\frac{2}{3}$ ). Proof (Theorem 3) Let $s$ be an arbitrary strategy profile and let $s^{*}$ be a strategy profile that minimizes $C$. We can assume without loss of generality that $d_{e}(x)=x$ for all $e \in E$ (see, e.g., [26]).

Recall that the social cost is defined as $C(s)=\sum_{i=1}^{n} c_{i}(s)$. The smoothness condition (2) is thus equivalent to

$$
\sum_{i=1}^{n}\left(c_{i}\left(s_{i}^{*}, s_{-i}\right)+\sum_{j \neq i} \alpha_{i j}\left(c_{j}\left(s_{i}^{*}, s_{-i}\right)-c_{j}(s)\right)\right) \leq \lambda C\left(s^{*}\right)+\mu C(s) .
$$

Let $x_{e}$ and $x_{e}^{*}$ refer to $x_{e}(s)$ and $x_{e}\left(s^{*}\right)$, respectively. Fix some player $i \in N$ and let $x_{e}^{\prime}=x_{e}\left(s_{i}^{*}, s_{-i}\right)$. Note that

$$
x_{e}^{\prime}= \begin{cases}x_{e}+1 & \text { if } e \in s_{i}^{*} \backslash s_{i} \\ x_{e}-1 & \text { if } e \in s_{i} \backslash s_{i}^{*} \\ x_{e} & \text { otherwise }\end{cases}
$$

Using these relations, we obtain

$$
\begin{aligned}
c_{i}\left(s_{i}^{*}, s_{-i}\right)+\sum_{j \neq i} \alpha_{i j}\left(c_{j}\left(s_{i}^{*}, s_{-i}\right)-c_{j}(s)\right) \\
=\sum_{e \in s_{i}^{*}} x_{e}^{\prime}+\sum_{j \neq i} \alpha_{i j} \sum_{e \in s_{j}}\left(x_{e}^{\prime}-x_{e}\right) \\
=\sum_{e \in s_{i}^{*}}\left(1+\sum_{j \neq i: e \in s_{j}} 1\right)+\sum_{j \neq i} \alpha_{i j}\left(\sum_{e \in s_{i}^{*} \cap s_{j}} 1-\sum_{e \in s_{i} \cap s_{j}} 1\right) \\
=\left|s_{i}^{*}\right|+\sum_{j \neq i}\left(\left(1+\alpha_{i j}\right)\left|s_{i}^{*} \cap s_{j}\right|-\alpha_{i j}\left|s_{i} \cap s_{j}\right|\right) .
\end{aligned}
$$

Summing over all players $i$, we obtain

$$
\begin{aligned}
& \sum_{i=1}^{n}\left(\left|s_{i}^{*}\right|+\sum_{j \neq i}\left(\left(1+\alpha_{i j}\right)\left|s_{i}^{*} \cap s_{j}\right|-\alpha_{i j}\left|s_{i} \cap s_{j}\right|\right)\right) \\
& \quad=\sum_{e \in E}\left(\sum_{i: e \in s_{i}^{*}} 1+\sum_{i: e \in s_{i}^{*}} \sum_{j \neq i: e \in s_{j}}\left(1+\alpha_{i j}\right)-\sum_{i: e \in s_{i}} \sum_{j \neq i: e \in s_{j}} \alpha_{i j}\right) \\
& \quad \leq \sum_{e \in E}\left(x_{e}^{*}+2 x_{e}^{*} x_{e}\right),
\end{aligned}
$$

where the inequality holds because the altruistic social context is restricted, i.e., $0 \leq \alpha_{i j} \leq 1$ for every $i, j \in N, i \neq j$. Applying Lemma 1 (with $x=x_{e}$ and $y=x_{e}^{*}$ ), we bound

$$
\sum_{e \in E}\left(x_{e}^{*}+2 x_{e}^{*} x_{e}\right) \leq \frac{7}{3} C\left(s^{*}\right)+\frac{2}{3} C(s),
$$

which concludes the proof. 
Unrestricted altruistic social context. Theorem 3 considers congestion games with restricted altruistic social context $\alpha$. This assumption is required; we now present an example showing that the price of anarchy is unbounded if the altruistic social context is unbounded.

Example 1 Let $\left(N, E,\left\{d_{e}\right\}_{e \in E},\left\{\Sigma_{i}\right\}_{i \in N}\right\}$ be a congestion game with $N=$ $\{1,2\}$ and $E=\{1,2,3,4\}$. Let the delay functions be defined by $d_{1}(x)=$ $d_{3}(x)=x$ and $d_{2}(x)=d_{4}(x)=K x$ for all $x$, where $K$ is a large constant. The strategy sets are $\Sigma_{1}=\{\{1,2\},\{3\}\}, \Sigma_{2}=\{\{3,4\},\{1\}\}$. Suppose furthermore that $\alpha$ is given by $\alpha_{11}=\alpha_{22}=0$ and $\alpha_{12}=\alpha_{21}=1$.

Then observe that in the $\alpha$-altruistic extension of this game, the strategy profile $(\{1,2\},\{3,4\})$ is a pure Nash equilibrium with social cost $2 K+2$. The optimal social cost is 2 , and is attained by the strategy profile $(\{3\},\{1\})$. The price of anarchy in this game is therefore $K+1$, and $K$ can be taken arbitrarily large, so the price of anarchy is unbounded.

\subsection{Load Balancing Games}

We derive a better smoothness result for singleton congestion games with identical delay functions, i.e., when $\Sigma_{i} \subseteq E$ for every $i \in N$ so that for each strategy $s \in \Sigma_{i}$ we have that $|s|=1$ and $\bar{d}_{e}(x)=x$ for every facility $e \in E$. We also refer to these games as load balancing games below.

Theorem 4 Every $\alpha$-altruistic extension of a load balancing game under restricted altruistic social context $\alpha$ is $\left(1+\varphi, 1 / \varphi^{2}\right)$-smooth, where $\varphi=(1+\sqrt{5}) / 2$ is the golden ratio. Therefore, the coarse correlated price of anarchy is at most $\varphi^{3} \approx 4.236$ for these games.

To proof Theorem 4 we need the following lemma, which is a variation of Lemma 1 in [16]. Its proof can be found in Appendix B.

Lemma 2 Let $\varphi=\frac{1+\sqrt{5}}{2}$ be the golden ratio. For every two integers $x, y \in \mathbb{N}$

$$
2 x y-\varphi x+\varphi^{2} y \leq \varphi^{2} y^{2}+\frac{1}{\varphi^{2}} x^{2} .
$$

Proof (Theorem 4) As in the proof of Theorem 3, it is sufficient to show that (3) holds with $(\lambda, \mu)=\left(1+\varphi, 1 / \varphi^{2}\right)$. Recall that $\varphi$ is the golden ratio and thus $\lambda=1+\varphi=\varphi^{2}$.

Following the same line of arguments as in the proof of Theorem 3, we obtain from (4) that we need to show that

$$
\sum_{e \in E}\left(x_{e}^{*}+2 x_{e}^{*} x_{e}\right) \leq \varphi^{2} C\left(s^{*}\right)+\frac{1}{\varphi^{2}} C(s) .
$$

The crucial insight here is that for load balancing games we have $\sum_{e \in E} x_{e}^{*}=$ $\sum_{e \in E} x_{e}=n$. We can thus rewrite the summation on the lefthand side above

$$
\sum_{e \in E}\left(2 x_{e}^{*} x_{e}-(\lambda-1) x_{e}+\lambda x_{e}^{*}\right)=\sum_{e \in E}\left(2 x_{e}^{*} x_{e}-\varphi x_{e}+\varphi^{2} x_{e}^{*}\right),
$$


by the definition of $\lambda$. Applying Lemma 2 (with $x=x_{e}$ and $y=x_{e}^{*}$ for each e) shows (5).

\section{Minsum Machine Scheduling}

In a scheduling game, we deal with a set of machines $[m]$, and a set of jobs $[n]$ that are to be scheduled on the machines. For each job $i \in[n]$ and machine $k \in[m]$, we are given a processing time $p_{i, k} \in \mathbb{R}_{\geq 0}$, which is the time it takes to run job $i$ on machine $k$.

There are many ways in which a machine may execute the set of jobs it gets assigned. We restrict ourselves here to a popular policy where the jobs on a machine are executed one-by-one, in order of increasing processing time (i.e., the longer jobs are executed later). Ties are broken in a deterministic way, and we write $i \prec_{k} j$ if $p_{i, k}<p_{j, k}$ or $p_{i, k}=p_{j, k}$ and the tie breaking rule schedules job $i$ before job $j$ on machine $k$. A schedule is a vector $s=\left(s_{1}, \ldots, s_{n}\right)$, where for $i \in[n], s_{i}$ is the machine on which job $i$ is to be run. We define the value $N(i, k, s)$ to be the number of jobs $j$ on machine $k$ under strategy profile $s$ for which it holds that $i \prec_{k} j$. Given $s$, the completion time of a job $i$ under $s$ is

$$
p_{i, s_{i}}+\sum_{j: j \prec_{s_{i}} i, s_{j}=s_{i}} p_{j, s_{j}} .
$$

The jobs take the role of the players: the strategy set of a player is $[m]$, so the strategy profiles that arise are schedules. The $\operatorname{cost} c_{j}(s)$ of a job $j \in[n]$ under strategy profile $s$ is the completion time of $j$ under $s$.

We define the social cost function for this game to be the sum of the completion times of the jobs. Note that the social cost can be written as

$$
C(s)=\sum_{k=1}^{m} \sum_{i: s_{i}=k}(N(i, k, s)+1) p_{i, k} .
$$

If the processing times are not restricted in any way, we speak of unrelated machine scheduling games. We speak of related machine scheduling games if the processing times are defined as follows: For each machine $k \in[m]$, there is a speed $t_{k} \in \mathbb{R}_{>0}$ and for each job $j \in[n]$ there is a length $p_{j} \in \mathbb{R}_{\geq 0}$ such that $p_{i, k}=p_{j} / t_{k}$ for all $i \in[n], k \in[m]$.

Cole et al. [17] show that unrelated machine scheduling games (without altruism) are $(2,1 / 2)$-smooth, resulting in a coarse correlated price of anarchy of at most $4 .^{2}$ Hoeksma and Uetz [25] prove that related machine scheduling games (without altruism) are $(2,0)$-smooth, leading to the conclusion that the coarse correlated price of anarchy is at most 2 .

\footnotetext{
2 More precisely, this is shown to hold for the more general case when the social cost is an arbitrary nonnegative linear combination of the player's cost. From a scheduling game instance described in [18], it follows that this bound is tight, i.e., that the coarse correlated price of anarchy is actually exactly 4 .
} 
Next, we prove constant upper bounds on the price of anarchy for restricted altruistic social contexts.

Theorem 5 Every $\alpha$-altruistic extension of a machine scheduling game with restricted altruistic social context $\alpha$ is $(2+x, 1 / x)$-smooth for related machines and $(2+x, 1 / 2+1 / x)$-smooth for unrelated machines for all $x \in \mathbb{R}$ such that $x \geq 1$ and $x \geq 2$ respectively. Therefore, the coarse correlated price of anarchy is at most $4+2 \sqrt{3} \approx 7.4641$ (choosing $x=1+\sqrt{3}$ ) and $12+8 \sqrt{2} \approx 23.3137$ (choosing $x=2+2 \sqrt{2}$ ) for these games, respectively.

Proof Recall that we assume that $\alpha$ is a restricted social context such that $\alpha_{i i}=1$ for all $i \in[n]$ and $\alpha_{i j} \in[0,1]$ for all $i, j \in[n]$.

In [25] it is proven that the base game for the case of related machines is $(2,0)$-smooth, and from the proof of Theorem 3.2 in [17], it follows that the base game for the case of unrelated machines is $(2,1 / 2)$-smooth. Thus, let $s^{*}$ be an arbitrary optimal schedule and let $s$ be an arbitrary schedule. It suffices to show that

$$
\sum_{i=1}^{n} \sum_{j \in[n]: j \neq i} \alpha_{i j}\left(c_{j}\left(s_{i}^{*}, s_{-i}\right)-c_{j}(s)\right) \leq x C\left(s^{*}\right)+\frac{C(s)}{x}
$$

for all $x>0$.

Let

$$
\begin{aligned}
& P_{1}=\left\{(i, j): s_{i}^{*}=s_{j}, s_{i}^{*} \neq s_{i}, i \prec_{s_{i}^{*}} j\right\}, \quad \text { and } \\
& P_{2}=\left\{(i, j): s_{i}=s_{j}, s_{i}^{*} \neq s_{j}, i \prec_{s_{i}} j\right\} .
\end{aligned}
$$

Informally, $P_{1}\left(P_{2}\right)$ is the set of pairs of jobs $(i, j)$ such that $i$ 's strategy change from $s_{i}$ to $s_{i}^{*}$ makes $j$ become scheduled later (earlier), for jobs $j$ that are scheduled on machine $s_{i}^{*}$ under $s$ (on machine $s_{i}$ under $s$ ). Note that for a pair of players $(i, j)$ that is not in $P_{1} \cup P_{2}$, we have $c_{j}\left(s_{i}^{*}, s_{-i}\right)-c_{j}(s)=0$, and for $(i, j) \in P_{2}$ it holds that $\alpha_{i j}\left(c_{j}\left(s_{i}^{*}, s_{-i}\right)-c_{j}(s)\right) \leq 0$.

Therefore:

$$
\begin{aligned}
\sum_{i=1}^{n} & \sum_{j \in[n]: j \neq i} \alpha_{i j}\left(c_{j}\left(s_{i}^{*}, s_{-i}\right)-c_{j}(s)\right)=\sum_{(i, j) \in P_{1} \cup P_{2}} \alpha_{i j}\left(c_{j}\left(s_{i}^{*}, s_{-i}\right)-c_{j}(s)\right) \\
& \leq \sum_{(i, j) \in P_{1}} \alpha_{i j}\left(c_{j}\left(s_{i}^{*}, s_{-i}\right)-c_{j}(s)\right) \leq \sum_{(i, j) \in P_{1}}\left(c_{j}\left(s_{i}^{*}, s_{-i}\right)-c_{j}(s)\right) \\
& =\sum_{(i, j) \in P_{1}} p_{i, s_{i}^{*}} .
\end{aligned}
$$

We now rewrite this last expression into a summation over the machines. We obtain:

$$
\sum_{i=1}^{n} \sum_{j \in[n]: j \neq i} \alpha_{i j}\left(c_{j}\left(s_{i}^{*}, s_{-i}\right)-c_{j}(s)\right)
$$




$$
\begin{aligned}
& \leq \sum_{k=1}^{m} \sum_{(i, j) \in P_{1}: s_{i}^{*}=k} p_{i, k} \\
& =\sum_{k=1}^{m} \sum_{i \in[n]: s_{i}^{*}=k} \sum_{j \in[n]:(i, j) \in P_{1}} p_{i, k} \\
& =\sum_{k=1}^{m} \sum_{\substack{i \in[n]: s_{i}^{*}=k, j \\
s_{i} \neq k}} \sum_{s_{j}=k, i \prec_{k} j} p_{i, k} \\
& =\sum_{k=1}^{m} \sum_{\substack{i \in[n]: s_{i}^{*}=k, s_{i} \neq k}} N(i, k, s) p_{i, k} \\
& =\sum_{k=1}^{m} \sum_{\substack{i \in[n]: s_{i}^{*}=k, s_{i} \neq k}}\left(x N\left(i, k, s^{*}\right)+x-1+N(i, k, s)-x N\left(i, k, s^{*}\right)-x+1\right) p_{i, k} \\
& \leq \sum_{k=1}^{m} \sum_{i \in[n]: s_{i}^{*}=k}\left(x\left(N\left(i, k, s^{*}\right)+1\right)-1\right) p_{i, k} \\
& +\sum_{k=1}^{m} \sum_{\substack{i \in[n]: s_{i}^{*}=k, s_{i} \neq k}}\left(N(i, k, s)-x N\left(i, k, s^{*}\right)-x+1\right) p_{i, k} \\
& \leq \sum_{k=1}^{m} \sum_{i \in[n]: s_{i}^{*}=k}\left(x\left(N\left(i, k, s^{*}\right)+1\right)-1\right) p_{i, k} \\
& +\sum_{k=1}^{m} \sum_{\substack{i \in[n]: s_{i}^{*}=k, s_{i} \neq k, N(i, k, s)>x N\left(i, k, s^{*}\right)+x-1}}\left\lceil N(i, k, s)-x N\left(i, k, s^{*}\right)-x+1\right\rceil p_{i, k} .
\end{aligned}
$$

Consider a job $i$ and machine $k$ such that it holds that $s_{i}^{*}=k, s_{i} \neq k$, and $N(i, k, s)>x N\left(i, k, s^{*}\right)+x-1$. Let $S(i, k)$ be the set of $\lceil N(i, k, s)-$ $\left.x N\left(i, k, s^{*}\right)-x\right\rceil$ smallest jobs $j \succ_{k} i$ such that $s_{j}=k$. Note that $S(i, k)$ is well defined in the sense that this number of jobs exists because $N(i, k, s)>$ $x N\left(i, k, s^{*}\right)+x-1$ implies $\left\lceil N(i, k, s)-x N\left(i, k, s^{*}\right)-x\right\rceil \geq 0$, and because there exist $N(i, k, s) \geq|S(i, k)|$ jobs $j \succ_{k} i$ with $s_{j}=k$. The the last expression equals

$$
\begin{aligned}
& \sum_{k=1}^{m} \sum_{i \in[n]: s_{i}^{*}=k}\left(x\left(N\left(i, k, s^{*}\right)+1\right)-1\right) p_{i, k} \\
& +\sum_{k=1}^{m} \sum_{\substack{i \in[n]: s_{i}^{*}=k, s_{i} \neq k, N(i, k, s)>x N\left(i, k, s^{*}\right)+x-1}}\left(p_{i, k}+\sum_{j \in S(i, k)} p_{i, k}\right),
\end{aligned}
$$


which is less than or equal to

$$
x C\left(s^{*}\right)+\sum_{k=1}^{m} \sum_{\substack{i \in[n]: s_{i}^{*}=k, s_{i} \neq k, N(i, k, s)>x N\left(i, k, s^{*}\right)+x-1}} \sum_{j \in S(i, k)} p_{i, k},
$$

thus, obtaining,

$$
\begin{aligned}
& \sum_{i=1}^{n} \sum_{j \in[n]: j \neq i} \alpha_{i j}\left(c_{j}\left(s_{i}^{*}, s_{-i}\right)-c_{j}(s)\right) \\
& \leq x C\left(s^{*}\right)+\sum_{k=1}^{m} \sum_{j \in[n]} \sum_{\substack{i \in[n]: j \in S(i, k), s_{i}^{*}=k, s_{i} \neq k, N(i, k, s)>x N\left(i, k, s^{*}\right)+x-1}} p_{i, k} .
\end{aligned}
$$

Note that for every job $j \in S(i, k)$ it holds that $N(j, k, s) \geq N(i, k, s)-$ $|S(i, k)|>x N\left(i, k, s^{*}\right)+x-1$. Therefore, the expression on the right-hand side of $(6)$ is equivalent to

$$
x C\left(s^{*}\right)+\sum_{k=1}^{m} \sum_{j \in[n]} \sum_{\begin{array}{c}
i \in[n]: j \in S(i, k), \\
s_{i}^{*}=k, \\
s_{i} \neq k, \\
N(i, k, s)>x N\left(i, k, s^{*}\right)+x-1 \\
N(j, k, s)>x N\left(i, k, s^{*}\right)+x-1
\end{array}} p_{i, k} .
$$

Note that $j \in S(i, k)$ implies that $s_{j}=k$ and that $i \prec_{k} j$. We relax some of the constraints in the summations and so we upper bound the expression in (7) by

$$
\begin{aligned}
& x C\left(s^{*}\right)+\sum_{k=1}^{m} \sum_{j \in[n]: s_{j}=k} \sum_{\substack{i \in[n]: s_{i}^{*}=k, s_{i} \neq k, i \prec_{k} j,}} p_{i, k} \\
& N(j, k, s)>x N\left(i, k, s^{*}\right)+x-1 \\
& \leq x C\left(s^{*}\right)+\sum_{k=1}^{m} \sum_{j \in[n]: s_{j}=k} \sum_{\substack{i \in[n]: s_{i}^{*}=k, s_{i} \neq k, i \prec k j,}} p_{j, k}
\end{aligned}
$$

The next step in the derivation is made by observing that for each job $j$ and each machine $k$ such that $s_{j}=k$, there are at most $\lceil(N(j, k, s)-x+1) / x\rceil$ jobs $i \prec_{k} j$ such that $s_{i}^{*}=k, s_{i} \neq k$ and $N(j, k, s)>x N\left(i, k, s^{*}\right)+x-1$. To see this, assume for contradiction that there are more than $\lceil(N(j, k, s)-x+1) / x\rceil$ jobs $i \prec_{k} j$ such that $s_{i}^{*}=k, s_{i} \neq k$ and $N(j, k, s)>x N\left(i, k, s^{*}\right)+x-1$. Let $i$ be the $(\lceil(N(j, k, s)-x+1) / x\rceil+1)$-th largest job for which these three 
properties hold. Then, there are at least $\lceil(N(j, k, s)-x+1) / x\rceil$ jobs scheduled on machine $k$ that have these properties and that are scheduled after $i$ on machine $k$ under strategy $s^{*}$. Therefore, we have that $x N\left(i, k, s^{*}\right)+x-1 \geq$ $x\lceil(N(j, k, s)-x+1) / x\rceil+x-1 \geq N(j, k, s)$, which is a contradiction.

Using this observation together with (6), (7) and (8), we obtain

$$
\begin{aligned}
\sum_{i=1}^{n} & \sum_{j \in[n]: j \neq i} \alpha_{i j}\left(c_{j}\left(s_{i}^{*}, s_{-i}\right)-c_{j}(s)\right) \\
& \leq x C\left(s^{*}\right)+\sum_{k=1}^{m} \sum_{j \in[n]: s_{j}=k}\left\lceil\frac{N(j, k, s)-x+1}{x}\right] p_{j, k} \\
& \leq x C\left(s^{*}\right)+\sum_{k=1}^{m} \sum_{j \in[n]: s_{j}=k} \frac{1}{x}(N(j, k, s)+1) p_{j, k} \\
& =x C\left(s^{*}\right)+\frac{C(s)}{x}
\end{aligned}
$$

where for the second inequality we use the basic fact that $\lceil a\rceil \leq a+1$ for all $a \in \mathbb{R}$.

Similarly to congestion games, the assumption of the altruistic social context being restricted is necessary. Next we provide an example showing that the price of anarchy is unbounded if the altruistic social context is unrestricted.

Example 2 Fix a number $M \in \mathbb{R}_{>0}$ arbitrarily, and consider the scheduling game with two machines and two jobs. The speeds are given by $t_{1}=1 / M, t_{2}=$ 1. The job lengths are given by $p_{1}=p_{2}=1$. Suppose that the altruism levels are as follows: $\alpha_{11}=\alpha_{22}=\alpha_{21}=0, \alpha_{12}=1$. Then the schedule where job 1 is on machine 1 and job 2 is on machine 2 is a pure Nash equilibrium. The social cost of this equilibrium is $M+1$. When $M \geq 2$, it is a social optimum to schedule both jobs on machine 2 , and this schedule achieves a social cost of 3. Therefore, for $M \geq 2$, the price of anarchy of this altruistic scheduling game is $(M+1) / 3$. Because $M$ can be picked arbitrarily large, this shows that the price of anarchy is arbitrarily bad for altruistic extensions of scheduling games, when we allow arbitrary altruism levels.

\section{Profit Maximization Games and Generalized Second-Price Auctions}

In this section we study generalized second-price auctions, which are profit maximization games. We will, therefore, first extend our notions to profit maximization games. Then we will apply the extended framework to second-price auctions. 


\subsection{Profit Maximization Games}

The smoothness definition introduced in Section 2 can naturally be adapted to profit maximization games.

Let $\Gamma=\left(N,\left\{\Sigma_{i}\right\}_{i \in N},\left\{p_{i}\right\}_{i \in N}\right)$ be a strategic game, where a player $i$ seeks to maximize his direct profit function $p_{i}: \Sigma \rightarrow \mathbb{R}$. The $\alpha$-altruistic extension $\Gamma^{\alpha}$ of $\Gamma$ is defined similarly as for cost minimization games.

Definition 2 Let $\Gamma^{\alpha}$ be an $\alpha$-extension of a profit maximization game with $\alpha \in \mathbb{R}^{n \times n}$ and social welfare function $\Pi$. Further, let $s^{*}$ be a strategy profile that maximizes $\Pi$. $\Gamma^{\alpha}$ is $(\lambda, \mu)$-smooth if for every strategy profile $s \in \Sigma$ it holds that

$$
\sum_{i=1}^{n} \sum_{j=1}^{n} \alpha_{i j}\left(p_{j}\left(s_{i}^{*}, s_{-i}\right)-p_{j}(s)\right) \geq \lambda \Pi\left(s^{*}\right)-(\mu+1) \Pi(s) .
$$

The proof of the following theorem proceeds along the same lines as the one of Theorem 1 and is omitted.

Theorem 6 Let $\Gamma^{\alpha}$ be an $\alpha$-extension of a profit maximization game with $\alpha \in \mathbb{R}^{n \times n}$ and social welfare function $\Pi$. If $\Gamma^{\alpha}$ is $(\lambda, \mu)$-smooth with $\mu>-1$, then the coarse correlated price of anarchy of $\Gamma^{\alpha}$ is at most $(1+\mu) / \lambda$.

We are now ready to study generalized second-price auctions.

\subsection{Generalized Second-Price Auctions}

We study auctions where a set $N=[n]$ of $n$ bidders compete for $k$ slots. Each bidder $i \in N$ has a valuation $v_{i} \in \mathbb{R}_{\geq 0}$ and specifies a bid $b_{i} \in \mathbb{R}_{\geq 0}$. Each slot $j \in[k]$ has a click-through rate $\gamma_{j} \in \mathbb{R}_{\geq 0}$. Without loss of generality, we assume that the slots are sorted according to their click-through rates such that $\gamma_{1} \geq \cdots \geq \gamma_{k}$ and that $k=n .^{3}$

We consider the generalized second price auction (GSP) as the underlying mechanism. Given a bidding profile $b=\left(b_{1}, \ldots, b_{n}\right)$, GSP orders the bidders by nonincreasing bids and assigns them in this order to the slots. Each bidder pays the next highest bid for his slot. More precisely, let $b_{1} \geq \cdots \geq b_{n}$ be the ordered list of bids. We assume without loss of generality that if $b_{i}=b_{j}$ for two bidders $i>j$ then $i$ precedes $j$ in the order. Then bidder $i$ is assigned to slot $i$ and has to pay $b_{i+1}$, where we define $b_{n+1}=0$. The utility of player $i$ for bidding profile $b$ is defined as $u_{i}(b)=\gamma_{i}\left(v_{i}-b_{i+1}\right)$. The social welfare for a bidding profile $b$ is defined as $\Pi(b)=\sum_{i=1}^{n} \gamma_{i} v_{i}$.

A standard assumption in this setting is that bidders do not overbid their valuations, i.e., the strategy set of bidder $i$ is $\left[0, v_{i}\right]$ for all $i \in[n]$. This assumption is made for reasons related to individual rationality: overbidding is

\footnotetext{
3 If $k<n$ we can add $n-k$ dummy slots with click-through rate 0 ; if $k>n$ we can remove the $k-n$ last slots.
} 
a dominated strategy. The same reasoning still applies if players are altruistic. Therefore, we will assume that bidders do not overbid.

We now prove an upper bound of $O(n)$ on the coarse correlated price of anarchy of $\alpha$-altruistic extensions of generalized second price auctions if the altruistic social context is restricted.

Theorem 7 Every $\alpha$-altruistic extension of a generalized second price auction with restricted altruistic social context $\alpha$ is $\left(\frac{1}{2}, n\right)$-smooth. Therefore, the coarse correlated price of anarchy is at most $2(n+1)$ for these games.

Proof Let $b^{*}$ and $b$ be two bidding profiles. By renaming, we assume that for all $j$, bidder $j$ gets assigned to slot $j$ under bidding profile $b$.

The base game is known to be $\left(\lambda_{1}, \mu_{1}\right)=\left(\frac{1}{2}, 1\right)$-smooth [31]. That is, for every two bidding profiles $b, b^{*}$, it holds that $\sum_{i \in N} u_{i}\left(b_{i}^{*}, b_{-i}\right) \geq \frac{1}{2} \Pi\left(b^{*}\right)-\Pi(b)$.

It remains to bound

$$
\begin{aligned}
\sum_{i=1}^{n} \sum_{j \neq i} \alpha_{i j}\left(u_{j}\left(b_{i}^{*}, b_{-i}\right)-u_{j}(b)\right) & \geq \sum_{i=1}^{n} \sum_{j \neq i} \alpha_{i j}\left(-u_{j}(b)\right) \geq \sum_{i=1}^{n} \sum_{j \neq i} \alpha_{i j}\left(-\gamma_{j} v_{j}\right) \\
& \geq \sum_{i=1}^{n} \sum_{j \neq i}-\gamma_{j} v_{j} \geq-(n-1) \Pi(b) .
\end{aligned}
$$

Combining these inequalities proves $(\lambda, \mu)=\left(\frac{1}{2}, n\right)$-smoothness and applying Theorem 6 gives the result.

The following example shows that the $O(n)$ upper bound derived in Theorem 7 is asymptotically best possible if one sticks to the smoothness framework introduced above. As a consequence, in order to improve on our $O(n)$ bound one has to resort to more refined techniques.

Example 3 Let $n$ be even and define $k=n / 2$. We define an instance of $n+1$ bidders as follows. Let

$$
\gamma_{1}=\cdots=\gamma_{k}=1, \quad \gamma_{k+1}=\cdots=\gamma_{n}=\varepsilon \quad \text { and } \quad \gamma_{n+1}=\varepsilon^{2} .
$$

Subsequently, we will make sure that $\varepsilon, 0<\varepsilon<1$, is chosen sufficiently small. Further, define the bidder valuations as

$$
v_{1}=\cdots=v_{k-1}=\varepsilon, \quad v_{k}=\cdots=v_{n}=1 \quad \text { and } \quad v_{n+1}=0 .
$$

We assume that no bidder overbids his valuation. In particular, this implies that bidder $n+1$ bids zero always.

Let $b^{*}=\left(b_{1}^{*}, \ldots, b_{n+1}^{*}\right)$ be an optimal bidding profile maximizing social welfare. Note that $b_{i}^{*}>b_{n+1}^{*}=0$ for every $i \in\{1, \ldots, n\}$ because otherwise bidder $n+1$ would be assigned to a slot with click-through rate at least $\varepsilon$, which is a contradiction to the optimality of $b^{*}$. The total social welfare of $b^{*}$ is $\Pi\left(b^{*}\right)=k+\varepsilon+(k-1) \varepsilon^{2}$.

Fix a bidding profile $b=\left(b_{1}, \ldots, b_{n+1}\right)$ such that $b_{1}>\cdots>b_{n+1}=0$ and $b_{1}<b_{i}^{*}$ for every $i \in\{1, \ldots, n\}$. Note that this is always possible because 
$b_{i}^{*}>0$ for every $i \in\{1, \ldots, n\}$. Also note that this implies that $b_{i}<\varepsilon$ for all $i$ because we assume that bidders do not overbid. Thus, by choosing $\varepsilon$ sufficiently small, we ensure that the bids in $b$ become arbitrarily small but induce the order as indicated above. We have

$$
\Pi(b)=\sum_{i=1}^{n+1} \gamma_{i} v_{i}=(n-1) \varepsilon+1 .
$$

If bidder $i \in\{1, \ldots, n\}$ changes his bid from $b_{i}$ to $b_{i}^{*}$, then he is assigned to slot 1 in $\left(b_{i}^{*}, b_{-i}\right)$, thereby shifting the players $1, \ldots, i-1$ one slot down (relative to the slots they are assigned to under $b$ ). Observe that bidder $k$ is assigned to the last slot with click-through rate 1 . As a consequence, whenever one of the bidders $i \in\{k+1, \ldots, n\}$ deviates to $\left(b_{i}^{*}, b_{-i}\right)$, bidder $k$ is shifted from his slot $k$ with click-through rate 1 down to slot $k+1$ with click-through rate $\varepsilon$. We will exploit this below.

Consider the left-hand side of the smoothness definition

$$
\sum_{i=1}^{n+1} \sum_{j=1}^{n+1} \alpha_{i j}\left(u_{j}\left(b_{i}^{*}, b_{-i}\right)-u_{j}(b)\right) \text {. }
$$

Fix player $i$ and consider the contribution $\Delta_{j}(i)=\alpha_{i j}\left(u_{j}\left(b_{i}^{*}, b_{-i}\right)-u_{j}(b)\right)$ of player $j$ to the sum. Clearly, $\Delta_{j}(n+1)=0$ for every $j$ because bidder $n+1$ is assigned to slot $n+1$ under both profiles. Let $i \in\{1, \ldots, n\}$. We distinguish four cases depending on the position of $j$ with respect to $i$ :

Case $j>i$ : The deviation of $i$ does not affect player $j$ and thus $\Delta_{j}(i)=0$.

Case $j=i$ : Player $i$ moves up to the first slot for which he pays $b_{1}$. Thus

$$
\Delta_{i}(i)=\alpha_{i i}\left(\gamma_{1}\left(v_{i}-b_{1}\right)-\gamma_{i}\left(v_{i}-b_{i+1}\right)\right) .
$$

Case $j=i-1$ : Player $j$ moves down one slot for which he pays $b_{j+2}$ (instead of $b_{j+1}$ under $b$ ). Thus

$$
\Delta_{j}(i)=\alpha_{i j}\left(\gamma_{j+1}\left(v_{j}-b_{j+2}\right)-\gamma_{j}\left(v_{j}-b_{j+1}\right)\right) .
$$

Case $j<i-1$ : Player $j$ moves down one slot for which he pays $b_{j+1}$ (as under $b$ ). Thus

$$
\Delta_{j}(i)=\alpha_{i j}\left(\gamma_{j+1}\left(v_{j}-b_{j+1}\right)-\gamma_{j}\left(v_{j}-b_{j+1}\right)=\alpha_{i j}\left(\gamma_{j+1}-\gamma_{j}\right)\left(v_{j}-b_{j+1}\right) .\right.
$$

Choosing $\varepsilon$ sufficiently small we can make sure that the total contribution of the payments $b_{\ell}$ in each $\Delta_{j}(i)$ in each of the above four cases is negligible.

We consider the restricted social context, so without loss of generality we assume that all $\alpha_{i i}$ are normalized to 1 . Recall that $\gamma_{1}=1$. Ignoring the effect of the payments (which we just argued is negligible if we make $\varepsilon$ small enough), the total contribution to the left-hand side of the smoothness definition is thus

$$
\sum_{i=1}^{n}\left(\alpha_{i i}\left(\gamma_{1} v_{i}-\gamma_{i} v_{i}\right)+\sum_{j=1}^{i-1} \alpha_{i j}\left(\gamma_{j+1}-\gamma_{j}\right) v_{j}\right)
$$




$$
=\sum_{i=1}^{n}\left(1-\gamma_{i}\right) v_{i}+\sum_{i=1}^{n} \sum_{j=1}^{i-1} \alpha_{i j}\left(\gamma_{j+1}-\gamma_{j}\right) v_{j}
$$

Note that $\gamma_{j+1}-\gamma_{j}=0$ for all $j \neq k$ and $\gamma_{k+1}-\gamma_{k}=\varepsilon-1$. The above expression thus simplifies to

$$
\begin{aligned}
\sum_{i=k+1}^{n}(1-\varepsilon) v_{i}+\sum_{i=k+1}^{n} \alpha_{i k}\left(\gamma_{k+1}-\gamma_{k}\right) v_{k} & =k(1-\varepsilon)-(1-\varepsilon) \sum_{i=k+1}^{n} \alpha_{i k} \\
& =(1-\varepsilon)\left(k-\sum_{i=k+1}^{n} \alpha_{i k}\right) .
\end{aligned}
$$

By setting $\alpha_{i k}=1$ for every $i$, the above contribution is equal to zero.

To show $(\lambda, \mu)$-smoothness, we need to lower bound the latter expression by $\lambda \Pi\left(b^{*}\right)-(\mu+1) \Pi(b)$. That is, $\lambda$ and $\mu$ need to satisfy

$$
(1-\varepsilon)\left(k-\sum_{i=k+1}^{n} \alpha_{i k}\right)=0 \geq \lambda\left(k+\varepsilon+(k-1) \varepsilon^{2}\right)-(\mu+1)((n-1) \varepsilon+1) .
$$

which implies (letting $\varepsilon \rightarrow 0$ ) that $\mu+1 \geq \lambda k$. This provides an asymptotic lower bound of

$$
\frac{1+\mu}{\lambda} \geq \frac{k}{1}=\frac{n}{2}
$$

on the possible price of anarchy achievable by our smoothness framework.

\section{Conclusions}

We studied the coarse correlated price of anarchy of altruistic extensions of three fundamental classes of games. The main focus of this paper was put on deriving upper bounds that are independent of the underlying social network structure. An interesting open question is whether one can derive refined bounds by exploiting structural properties of the underlying social network.

In the present studies, we concentrated on altruistic games with nonnegative altruistic social contexts $\alpha$, even though our model of altruistic games and the smoothness definition introduced in Sections 2 and 3 allow us to incorporate spiteful behavior as well. We leave it as an interesting open direction for future research to pursue such analyses for spiteful behavior. Another open question to investigate is whether local smoothness conditions can be formulated such that they can be used for analysis of various altruistic games, in the spirit of [32].

\section{Acknowledgments}

We would like to thank Ludovic Amruthalingam (from the Institut fr Theoretische Informatik, ETH Zrich) for useful feedback on a preliminary version of this paper. We would like to thank the anonymous reviewers (both of TOCS and of the preliminary conference version [1]) for their valuable comments. 


\section{References}

1. Anagnostopoulos, A., Becchetti, L., de Keijzer, B., G. Schäfer, G.: Inefficiency of games with social context. In: Vöcking, B. (ed.) Proceedings of the 6th International Symposium on Algorithmic Game Theory, Lecture Notes in Computer Science, vol. 8146, pp. 219-230. Springer (2013)

2. Ashlagi, I., Krysta, P., Tennenholtz, M.: Social context games. In: Proceedings of the 4th International Workshop on Internet and Network Economics. pp. 675-683 (2008)

3. Awerbuch, B., Azar, Y., Epstein, A.: The price of routing unsplittable flow. In: Proceedings of the 37th ACM Symposium on Theory of Computing. pp. 57-66 (2005)

4. Bilò, V., Celi, A., Flammini, M., Gallotti, V.: Social context congestion games. In: Proceedings of the 18th international conference on Structural Information and Communication Complexity. pp. 282-293. Springer (2011)

5. Bilò, V., Fanelli, A., Flammini, M., Moscardelli, L.: Graphical congestion games. Algorithmica 61(2), 274-297 (2011)

6. Brandt, F., Sandholm, T., Shoham, Y.: Spiteful bidding in sealed-bid auctions. In: Proceedings of the 20th International Joint Conference on Artifical Intelligence. pp. 1207-1214 (2007)

7. Buehler, R., Goldman, Z., Liben-Nowell, D., Pei, Y., Quadri, J., Sharp, A., Taggart, S. Wexler, T., Woods, K.: The price of civil society. In: Proceedings of the 7 th International Workshop on Internet and Network Economics. pp. 375-382 (2011)

8. Caragiannis, I., Flammini, M., Kaklamanis, C., Kanellopoulos, P., Moscardelli, L.: Tight bounds for selfish and greedy load balancing. In: Automata, Languages and Programming, Lecture Notes in Computer Science, vol. 4051, pp. 311-322. Springer (2006)

9. Caragiannis, I., Kaklamanis, C., Kanellopoulos, P., Kyropoulou, M.: On the efficiency of equilibria in generalized second price auctions. In: Proceedings of the 12th ACM Conference on Electronic Commerce. pp. 81-90 (2011)

10. Caragiannis, I., Kaklamanis, C., Kanellopoulos, P., Kyropoulou, M., Papaioannou, E. The impact of altruism on the efficiency of atomic congestion games. In: Proceedings of the 5th Symposium on Trustworthy Global Computing (2010)

11. Caragiannis, I., Kaklamanis, C., Kanellopoulos, P., Kyropoulou, M., Lucier, B., Leme, R.P., Tardos, É.: On the efficiency of equilibria in generalized second price auctions. CoRR abs/1201.6429 (2012)

12. Chen, P.A., de Keijzer, B., Kempe, D., Schäfer, G.: The robust price of anarchy of altruistic games. In: Proceedings of the 7th International Workshop on Internet and Network Economics. pp. 383-390 (2011)

13. Chen, P.A., de Keijzer, B., Kempe, D., Schäfer, G.: Altruism and its impact on the price of anarchy. ACM Transactions on Economics and Computation (2014, to appear)

14. Chen, P.A., Kempe, D.: Altruism, selfishness, and spite in traffic routing. In: Proceedings of the 9th ACM Conference on Electronic Commerce. pp. 140-149 (2008)

15. Christodoulou, G., Koutsoupias, E.: The price of anarchy of finite congestion games. In: Proceedings of the 37th ACM Symposium on the Theory of Computing. pp. 67-73 (2005)

16. Christodoulou, G., Mirrokni, V.S., Sidiropoulos, A.: Convergence and approximation in potential games. Theoretical Computer Science 438(0), 13 -27 (2012)

17. Cole, R., Correa, J.R., Gkatzelis, V., Mirrokni, V., Olver, N.: Inner product spaces for minsum coordination mechanisms. In: Proceedings of the 43rd ACM Symposium on the Theory of Computing. pp. 539-548 (2011)

18. Correa, J.R., Queyranne, M.: Efficiency of equilibria in restricted uniform machine scheduling with total weighted completion time as social cost. Naval Research Logistics 59(5), 384-395 (2012)

19. Elias, J., Martignon, F., Avrachenkov, K., Neglia, G.: Socially-aware network design games. In: Proceedings of the 29th Conference on Computer Communications. pp. 4145 (2010)

20. Fehr, E., Schmidt, K.M.: The Economics of Fairness, Reciprocity and Altruism: Experimental Evidence and New Theories, Handbook on the Economics of Giving, Reciprocity and Altruism, vol. 1, chap. 8, pp. 615-691. Elsevier (2006) 
21. Fotakis, D., Gkatzelis, V., Kaporis, A.C., Spirakis, P.: The impact of social ignorance on weighted congestion games. In: Proceedings of the 5th International Workshop on Internet and Network Economics. pp. 316-327. Springer (2009)

22. Hoefer, M., Skopalik, A.: Altruism in atomic congestion games. Transactions on Economics and Computation 1(4), Article 21 (2009)

23. Hoefer, M., Skopalik, A.: Stability and convergence in selfish scheduling with altruistic agents. In: Proceedings of the 5th International Workshop on Internet and Network Economics. pp. 616-622 (2009)

24. Hoefer, M., Skopalik, A.: Social context in potential games. In: Proceedings of the 8th International Conference on Internet and Network Economics. pp. 364-377 (2012)

25. Hoeksma, R., Uetz, M.: The price of anarchy for minsum related machine scheduling. In: Proceedings of the 9th International Conference on Approximation and Online Algorithms. pp. 261-273 (2012)

26. de Keijzer, B.: Externalities and Cooperation in Algorithmic Game Theory. Ph.D. thesis, Centrum voor Wiskunde en Informatica (CWI) (2014)

27. Koutsoupias, E., Papadimitriou, C.: Worst-case equilibria. In: Proceedings of the 16th Symposium on Theoretical Aspects of Computer Science. pp. 404-413 (1999)

28. Leme, R.P., Tardos, É.: Pure and bayes-nash price of anarchy for generalized second price auction. In: 51th Annual IEEE Symposium on Foundations of Computer Science (FOCS). pp. 735-744 (2010)

29. Lucier, B., Leme, R.P.: GSP auctions with correlated types. In: Proceedings 12th ACM Conference on Electronic Commerce (EC). pp. 71-80 (2011)

30. Rahn, M., Schäfer, G.: Bounding the inefficiency of altruism through social contribution games. In: Chen, Y., Immorlica, N. (eds.) Proceedings of the 9th International Conference on Web and Internet Economics (WINE), Lecture Notes in Computer Science, vol. 8289, pp. 391-404. Springer (2013)

31. Roughgarden, T.: The price of anarchy in games of incomplete information. In: Proceedings of the 13th ACM Conference on Electronic Commerce. pp. 862-879 (2012)

32. Roughgarden, T., Schoppmann, F.: Local smoothness and the price of anarchy in atomic splittable congestion games. In: Proceedings of the 22nd ACM-SIAM Symposium on Discrete Algorithms. pp. 255-267 (2011)

33. Roughgarden, T.: Intrinsic robustness of the price of anarchy. Communication of the ACM 55(7), 116-123 (2012), (Preliminary version appeared in STOC 2009.)

34. Suri, S., Tóth, C.D., Zhou, Y.: Selfish load balancing and atomic congestion games. In: Proceedings of the Sixteenth Annual ACM Symposium on Parallelism in Algorithms and Architectures. pp. 188-195. ACM (2004)

35. Syrgkanis, V.: Bayesian games and the smoothness framework. CoRR abs/1203.5155 (2012)

36. Syrgkanis, V., Tardos, É.: Composable and efficient mechanisms. In: Proceedings of the 45th ACM Symposium on the Theory of Computing (2013)

37. Young, H.P.: Strategic Learning and its Limits. Oxford University Press (1995) 


\section{A Proofs of Theorem 1 and Theorem 2}

Proof (Theorem 1) Let $\sigma$ be a coarse correlated equilibrium for $\Gamma^{\alpha}$ and let $s$ be a random variable with distribution $\sigma$. Further, let $s^{*} \in \Sigma$ be an optimal strategy profile. The coarse correlated equilibrium condition implies that for every player $i \in N$ :

$$
\sum_{j=1}^{n} \alpha_{i j} \mathbf{E}\left[c_{j}\left(s_{i}^{*}, s_{-i}\right)\right]-\sum_{j=1}^{n} \alpha_{i j} \mathbf{E}\left[c_{j}(s)\right] \geq 0 .
$$

By summing over all players and using linearity of expectation, we obtain

$$
\mathbf{E}[C(s)] \leq \mathbf{E}[C(s)]+\mathbf{E}\left[\sum_{i=1}^{n} \sum_{j=1}^{n} \alpha_{i j}\left(c_{j}\left(s_{i}^{*}, s_{-i}\right)-c_{j}(s)\right)\right] .
$$

Now we use the smoothness property (2) and obtain

$$
\mathbf{E}[C(s)] \leq \mathbf{E}[C(s)]+\mathbf{E}\left[\lambda C\left(s^{*}\right)+(\mu-1) C(s)\right]=\lambda C\left(s^{*}\right)+\mu \mathbf{E}[C(s)] .
$$

Since $\mu<1$, this implies that the coarse correlated price of anarchy is at most $\lambda /(1-\mu)$.

Proof (Theorem 2) Consider a sequence $s^{1}, \ldots, s^{T}$ of outcomes of $\Gamma^{\alpha}$ that satisfies the vanishing average external regret property, i.e., for every player $i \in N$

$$
\frac{1}{T} \sum_{t=1}^{T} c_{i}^{\alpha}\left(s^{t}\right) \leq \frac{1}{T}\left[\min _{s_{i}^{\prime} \in \Sigma_{i}} \sum_{t=1}^{T} c_{i}^{\alpha}\left(s_{i}^{\prime}, s_{-i}^{t}\right)\right]+o(1) .
$$

Let $s^{*}$ be an optimal outcome that minimizes the social cost function $C$. Define

$$
\delta_{i}^{\alpha}\left(s^{t}\right)=c_{i}^{\alpha}\left(s^{t}\right)-c_{i}^{\alpha}\left(s_{i}^{*}, s_{-i}^{t}\right)
$$

for every $i \in N$ and $t \in\{1, \ldots, T\}$. Exploiting (10), we obtain that for every player $i \in N$

$$
\frac{1}{T} \sum_{t=1}^{T} \delta_{i}^{\alpha}\left(s^{t}\right) \leq \frac{1}{T}\left(\sum_{t=1}^{T} c_{i}^{\alpha}\left(s^{t}\right)-\min _{s_{i}^{\prime} \in \Sigma_{i}} \sum_{t=1}^{T} c_{i}^{\alpha}\left(s_{i}^{\prime}, s_{-i}^{t}\right)\right)=o(1) .
$$

Let $\Delta^{\alpha}\left(s^{t}\right)=\sum_{i=1}^{n} \delta_{i}^{\alpha}\left(s^{t}\right)$. We have

$$
\Delta^{\alpha}\left(s^{t}\right)=\sum_{i=1}^{n}\left(c_{i}^{\alpha}\left(s^{t}\right)-c_{i}^{\alpha}\left(s_{i}^{*}, s_{-i}^{t}\right)\right)=\sum_{i=1}^{n} \sum_{j=1}^{n} \alpha_{i j}\left(c_{j}\left(s^{t}\right)-c_{j}\left(s_{i}^{*}, s_{-i}^{t}\right)\right) .
$$

Exploiting that $\Gamma^{\alpha}$ is $(\lambda, \mu)$-smooth, we obtain

$$
C\left(s^{t}\right) \leq \frac{\lambda}{1-\mu} C\left(s^{*}\right)+\frac{\Delta^{\alpha}\left(s^{t}\right)}{1-\mu} .
$$

By summing (12) over all $t$ and using (11), we obtain that the average cost of the sequence of $T$ outcomes is

$$
\frac{1}{T} \sum_{t=1}^{T} C\left(s^{t}\right) \leq \frac{\lambda}{1-\mu} C\left(s^{*}\right)+\frac{1}{1-\mu} \sum_{i=1}^{n}\left(\frac{1}{T} \sum_{t=1}^{T} \delta_{i}^{\alpha}\left(s^{t}\right)\right) \stackrel{T \rightarrow \infty}{\longrightarrow} \frac{\lambda}{1-\mu} C\left(s^{*}\right) .
$$




\section{B Proof of Lemma 2}

Proof (Lemma 2) It is easy to check that the claim holds if $x=0$ or $y=0$. Let $x \geq 1$ and $y \geq 1$. Recall that $1+\varphi=\varphi^{2}$. We have

$$
\begin{aligned}
\varphi^{2} y^{2} & -2 x y+\frac{1}{\varphi^{2}} x^{2}+\varphi x-\varphi^{2} y=\left(\varphi y-\frac{1}{\varphi} x\right)^{2}+\varphi x-(1+\varphi) y \\
& \geq 2 \varphi y-\frac{2}{\varphi} x-1+\varphi x-(1+\varphi) y=(\varphi-1) y+\left(\varphi-\frac{2}{\varphi}\right) x-1 \\
& =\frac{1}{\varphi} y+\left(1-\frac{1}{\varphi}\right) x-1 \geq 0,
\end{aligned}
$$

where the first inequality follows because $z^{2} \geq 2 z-1$ for every $z \in \mathbb{R}$ and the last one holds because $x \geq 1$ and $y \geq 1$. 